\title{
Biblioteca Pública de Segovia
}

\author{
MARÍA LUISA SANTAMARÍA RAMÍREZ \\ Biblioteca Pública de Segovia
}

\begin{abstract}
La Biblioteca Pública de Segovia abrió sus puertas en el nuevo edificio en enero de 2016. A lo largo de su historia, iniciada en 1836 , ha pasado por vicisitudes y dificultades, alcanzando ahora una estabilidad propiciada por unas instalaciones adecuadas y una provisión de fondos ajustada a su gestión. Una biblioteca moderna al servicio de los ciudadanos y sus necesidades.
\end{abstract}

Bibliotecas públicas, historia de las bibliotecas, estadísticas de usuarios, fomento de la lectura, redes de bibliotecas

\section{Introducción}

La Biblioteca Pública de Segovia es un centro perteneciente a la red de bibliotecas de Castilla y León, dependiente de la Consejería de Cultura y Turismo de la Junta de Castilla y León. Es la biblioteca cabecera del sistema de la provincial, lo que significa que, además de cubrir las necesidades de información de la ciudad (hasta hace 4 años era la única biblioteca pública en Segovia), debe también prestar servicios y asesoramiento técnico a las bibliotecas integradas en la provincia.

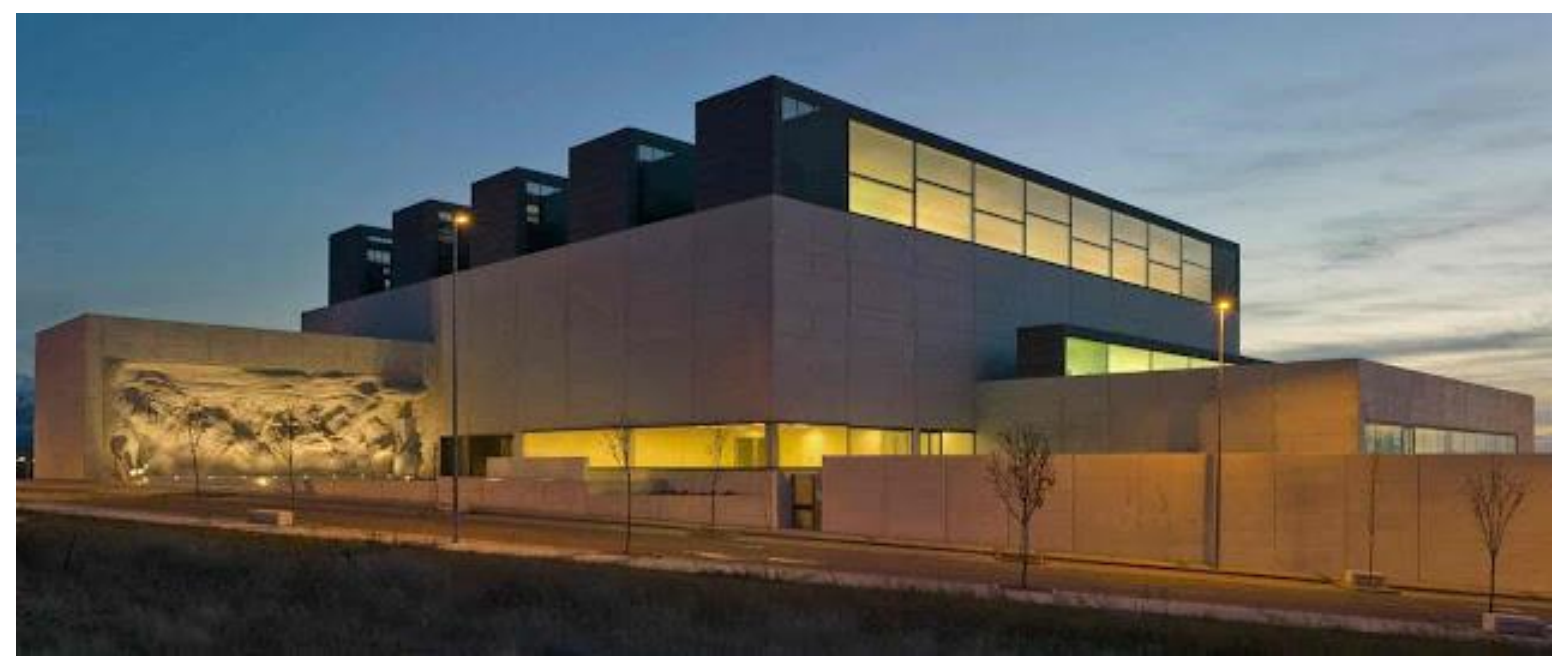

Figura 1: Imagen nocturna de la biblioteca. (Foto: Cano y Escario Arquitectos)

Bajo el principio de que el acceso abierto a los resultados de investigación acelera el avance del conocimiento, todos los contenidos de la edición electrónica de CLIP se distribuyen bajo una licencia de uso y distribución Creative Commons Reconocimiento-NoComercialCompartirlgual 3.0 España (CC BY-NC-SA 3.0 ES). 
La biblioteca es de titularidad estatal, transferida su gestión a la Junta de Castilla y León en 1986 mediante convenio, siendo la Consejería de Cultura y Turismo quien se ocupa de dotarla de presupuesto para la adquisición de fondo bibliográfico, reparación y mantenimiento de los edificios, así como la celebración de actividades culturales, además de la gestión del personal y de la coordinación de programas de diversa índole.

Está regulada por el Real Decreto 582/1989, de 19 de mayo, en el que se aprueba el Reglamento de Bibliotecas Públicas del Estado y del Sistema Español de Bibliotecas y por el Decreto 263/1997, de 26 de diciembre, por el que se aprueba el Reglamento interno de organización de los servicios de las Bibliotecas Públicas de titularidad estatal gestionadas por la Comunidad de Castilla y León, donde se establecen los servicios que han de prestar, horarios, materiales de las colecciones, acceso a los fondos, su participación en los planes cooperativos del Sistema de Bibliotecas de Castilla y León, etc. Además, en la carta de servicios publicada en la Orden $\mathrm{ADM} / 745 / 2008$, de 18 de abril se establecen sus funciones, competencias y servicios:

Funciones y competencias

- La Biblioteca Pública de Segovia tiene como misión facilitar a los ciudadanos el acceso a la información, a la cultura y a la formación permanente en el contexto de la Sociedad del Conocimiento, poniendo a su disposición una colección organizada de materiales bibliográficos, audiovisuales y digitales, así como de otros recursos de información.

- La Biblioteca asume un compromiso especial de servicio a la comunidad más cercana, elaborando y difundiendo información de interés para esta y manteniendo una amplia colección de libros y otros recursos de información de temas y autores locales y provinciales.

- Tiene como función la coordinación bibliotecaria del sistema provincial y el asesoramiento a las bibliotecas integradas en el mismo, promoviendo la cooperación y el trabajo en red entre éstas.

- Le corresponde incrementar, preservar y difundir los bienes integrantes del Patrimonio Bibliográfico Español y de Castilla y León que se custodian en sus instalaciones, incluidos los fondos depositados en la biblioteca en aplicación de la normativa de Depósito Legal.

Servicios que se ofrece

La Biblioteca Pública de Segovia ofrece sus servicios y sus recursos, en igualdad de oportunidades y sin discriminación por causa alguna, a todas las personas, colectivos y entidades que los soliciten. Sus servicios fundamentales son los siguientes:

1. Préstamo personal o colectivo de libros, revistas, audiovisuales y recursos digitales.

2. Lectura y consulta de las colecciones impresas, audiovisuales y digitales de la biblioteca.

3. Información y respuesta a las consultas de los usuarios sobre temas de carácter general, especializado y local.

4. Programas formativos para facilitar a los usuarios el conocimiento y uso de la biblioteca y el acceso a los recursos de información.

5. Disponibilidad de equipos y programas informáticos y de acceso a Internet.

6. Actividades culturales y programas de promoción de la lectura y desarrollo de los hábitos lectores.

7. Asistencia técnica y asesoramiento a bibliotecas, colectivos e instituciones para la organización de servicios bibliotecarios y de información. 


\section{Historia}

La Biblioteca Pública de Segovia tiene su origen en la desamortización de las bibliotecas conventuales de Segovia y provincia, muchas de ellas maltrechas tras el paso de las tropas francesas. Estos fondos pasaron por diversas localizaciones y mermas desde 1836, hasta que comenzó su andadura en junio de 1842 con 4150 volúmenes, convirtiéndose en la primera biblioteca pública provincial en abrir sus puertas, bajo la dirección de Felipe Pardo ayudado por el bibliotecario Santos Muñoz, en la capilla y sacristía del Hospital de viejos, situado en el palacio de Enrique IV. Este primer lote fundacional comienza a incrementarse con la compra de otros documentos adquiridos con el dinero que proporcionaba la venta por arrobas de los libros en mal estado o de escaso interés y con donaciones.

Desde 1886 hasta 1934, la biblioteca estuvo ubicada en el Instituto Provincial de Segunda Enseñanza situado en el edificio de la Fundación Ondátegui, pasando después a las instalaciones de San Quirce, sede de la Universidad Popular, donde estuvo hasta 1948. A partir de este año, la biblioteca tomó posesión del edificio conocido como Cárcel vieja, antaño convento, compartiéndolo con el Museo Provincial (hasta 1955) y con el Archivo Provincial (hasta 1996). En este periodo, su directora fue Manuela Villalpando, primera mujer académica de San Quirce.

La biblioteca en este edificio tuvo que pasar por varios periodos de obras para acondicionarlo y posibilitar la ampliación de espacios que acogieran los fondos. Sin embargo, al tratarse de un edificio con unas características tan específicas en cuanto a la disposición de estancias, era muy difícil albergar en él la colección, así como ofrecer los servicios que los nuevos tiempos incorporaban a las bibliotecas. Viendo la necesidad de ello, el Ministerio de Cultura construyó un nuevo edificio, moderno, amplio y accesible, que fue inaugurado en enero de 2016, siendo Director Luis García Méndez. A su jubilación, en 2017, le sucedió en el puesto Roberto García Arribas, actual Director del centro.

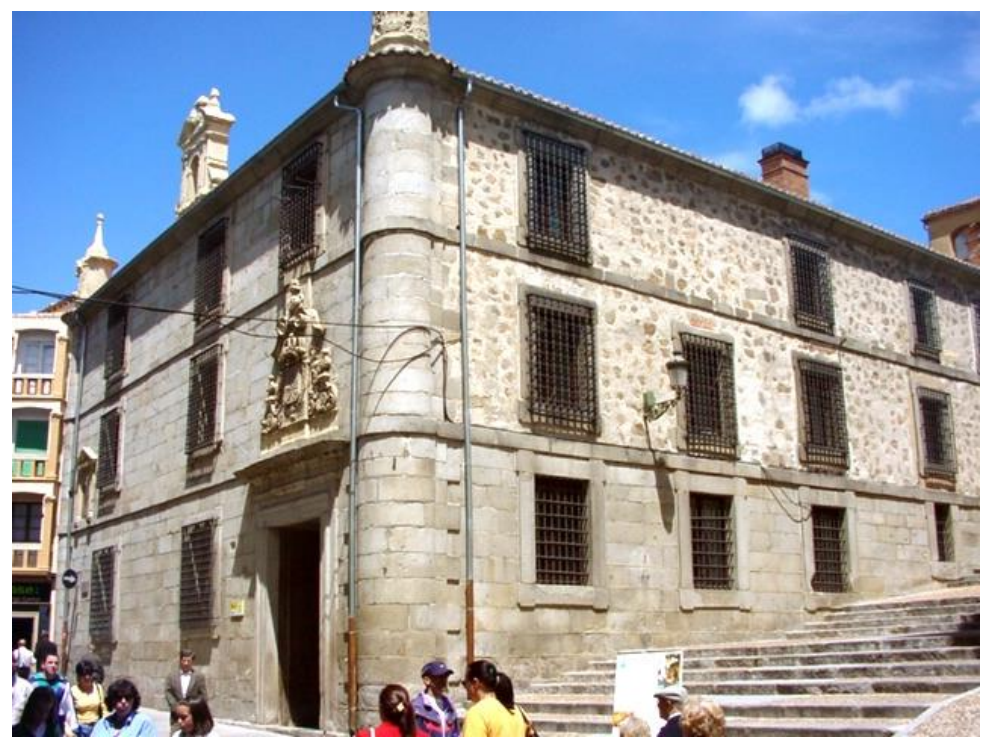

Figura 2: Edificio de la Cárcel vieja (Foto: archivo del Ayuntamiento de Segovia) 
El edificio fue diseñado por Benjamín Cano, del estudio Cano y Escario Arquitectura, dotándolo de una gran singularidad por su estructura equipada con grandes ventanales desde donde contemplar el entorno de montaña y meseta que rodea la ciudad, así como por el diálogo entre pasado y presente con la incorporación para los suelos de piedra de la cantera cántabra de Tina Menor en la que es posible observar gran cantidad de fósiles marinos. Cuenta también con un mural diseñado por Cano, con relieves de manos que simbolizan el instante creador y la multiplicidad literaria.

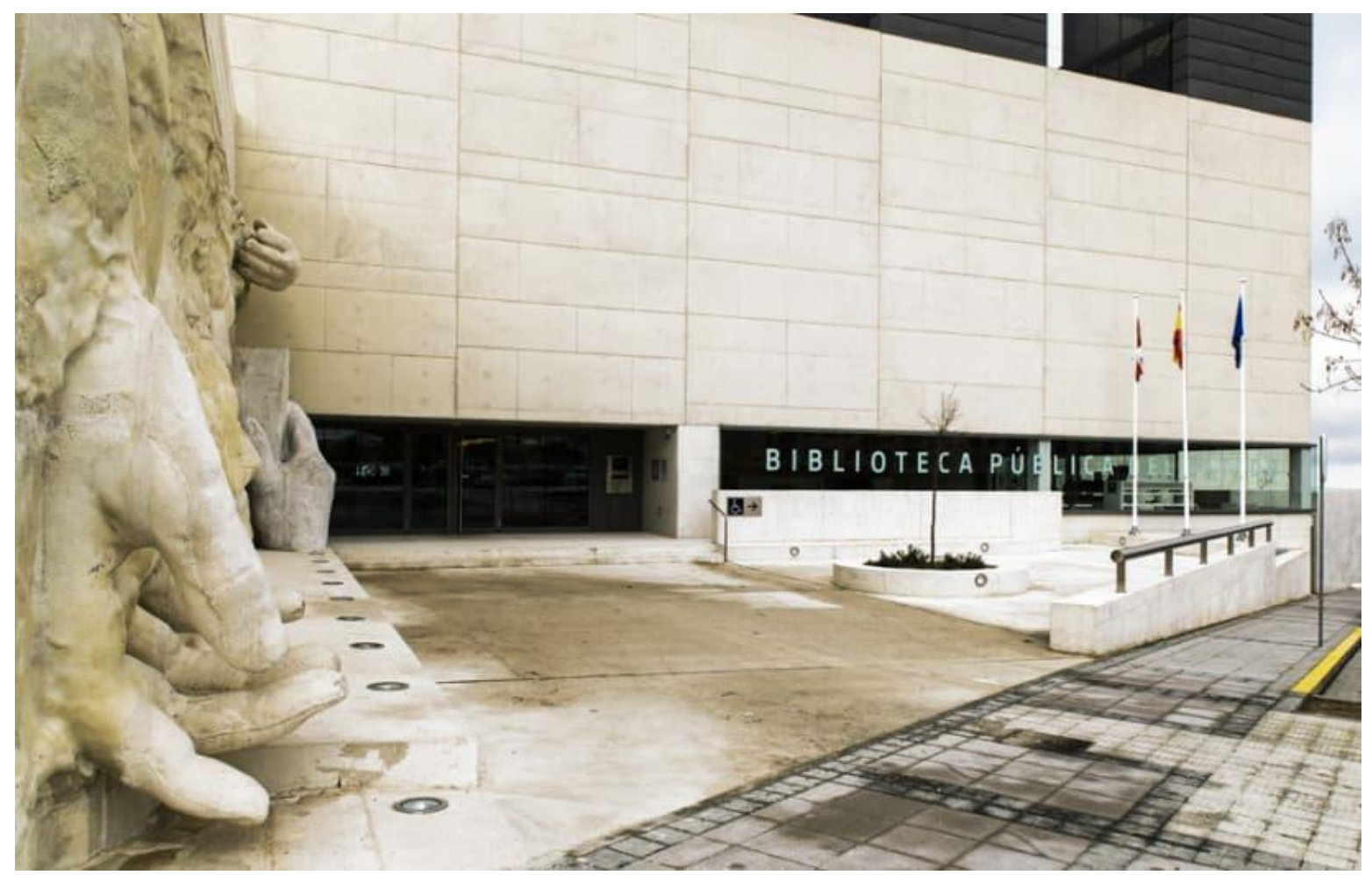

Figura 3: Entrada principal con mural (Foto: Cano y Escario Arquitectos)

RABEL

La Biblioteca Pública de Segovia pertenece a RABEL, Red Automatizada de Bibliotecas de Castilla y León, que se creó en 2009 y ha permitido la interconexión en red de la mayoría de las bibliotecas integradas en el Sistema de Bibliotecas de Castilla y León. Este servicio lo sufraga la Consejería de Cultura y Turismo y se coordina desde la Biblioteca de Castilla y León. Esto permite, entre otras cosas, la catalogación compartida y la implantación de una tarjeta de usuario única para toda la Comunidad que facilita a los usuarios el acceso a servicios y recursos de los centros integrantes de RABEL, para lo cual se han establecido diferentes modalidades de tarjetas de usuarios:

- Carné infantil-juvenil, para usuarios hasta 14 años.

- Carné de adulto, a partir de 14 años, con validez indefinida.

- Carné de investigador, para el acceso a colecciones especiales. 
- Carné de profesional de la documentación, para el acceso a colecciones especializadas de Biblioteconomía y Documentación.

- Carné de colectivo, para centros escolares, asociaciones e instituciones.

- Carné de clubes de lectura, para la gestión del préstamo de lotes de libros a clubes de lectura.

\section{La biblioteca en cifras}

Según cifras de 2019, la biblioteca cuenta con una colección de 186.255 documentos, repartidos de la siguiente manera:

\begin{tabular}{|c|c|c|c|c|}
\hline Libros & $\begin{array}{c}\text { P. } \\
\text { Periódicas } \\
\end{array}$ & Vídeos & $\begin{array}{l}\text { Discos } \\
\text { Casetes }\end{array}$ & $\begin{array}{l}\text { yocumentos } \\
\text { electrónicos }\end{array}$ \\
\hline 152.951 & 1.170 & 16.092 & 9.091 & 1.278 \\
\hline
\end{tabular}

Figura 4: La colección (Anuario estadístico de Castilla y León 2020)

El total de tarjetas de usuario es de 23.152, siendo los usuarios activos 8.004, que contabilizan 114.608 préstamos:

\begin{tabular}{|c|c|c|c|c|c|}
\hline \multicolumn{3}{|c|}{ Usuarios } & \multicolumn{3}{|c|}{ Servicio de préstamo } \\
\hline Infantil & Adultos & Total & Infantil & Adultos & Total \\
\hline 5.643 & 26.509 & 32.152 & 22.564 & 92.044 & 114.608 \\
\hline
\end{tabular}

Figura 5: Tipos de usuarios y préstamos (Anuario estadístico de Castilla y León 2020)

Además, el número de visitantes a las instalaciones para el servicio de préstamo y participación en actividades durante el año 2019 fue de 203.692.

En ese mismo año, el número de actividades de animación lectora fue de 1026. En esta cifra se incluyen actividades tanto para usuarios infantiles, como juveniles y adultos: cuentacuentos, representaciones teatrales, conciertos, talleres, clubs de lectura, ciclos de literatura, de pensamiento, de ciencia, visitas guiadas... sin olvidar las actividades desarrolladas dentro del marco del programa Cultura Diversa de la Consejería de Cultura y Turismo. Este proyecto se puso en marcha en 2016, con el objetivo de garantizar el acceso a la cultura de todos los ciudadanos, especialmente de aquellos que tienen más dificultades por sus condiciones físicas, intelectuales o sociales: personas en situación de discapacidad, personas de la tercera edad, inmigrantes, desempleados, etc. Para ello, La Biblioteca Pública de Segovia colabora con asociaciones e 
instituciones como Cruz Roja, Caritas, Accem, Centro Penitenciario, Centros de mayores, Alzheimer Segovia y otras más para ofrecer actividades inclusivas que permitan a sus asociados conocer la biblioteca y lo que ofrece, así como darles visibilidad con respecto al resto de usuarios.

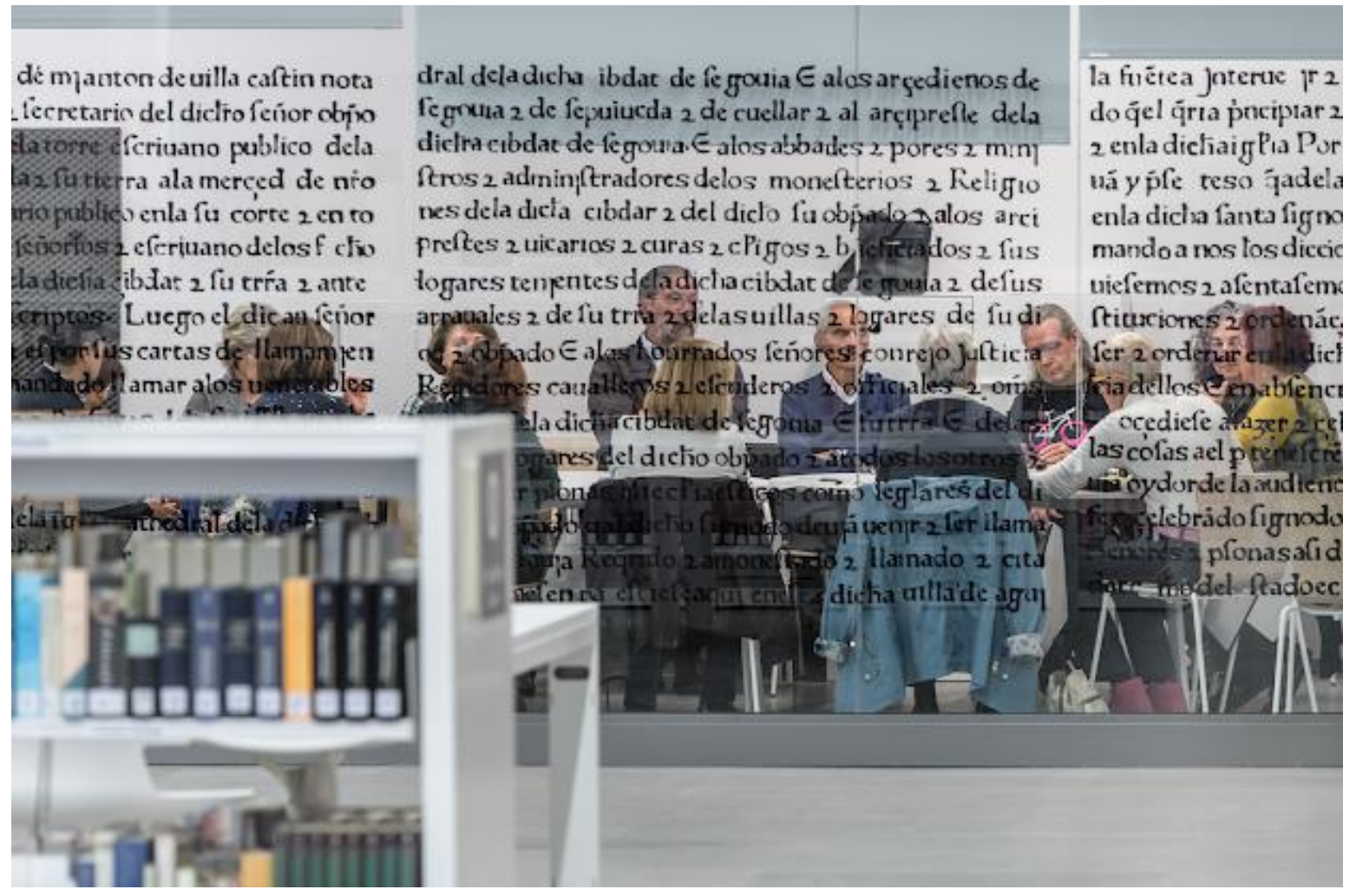

Figura 6: Sesión de club de lectura

\section{Secciones}

La biblioteca está organizada en diferentes secciones con espacios diferenciados: Préstamo de adultos, Consulta y Hemeroteca, Zona Joven, Sección Infantil, Sección Local y Fondo Antiguo.

Préstamo de adultos. Con una buena colección en formato libro y audiovisual, se trabaja constantemente en el mantenimiento y la ampliación de fondos a través de la adquisición de novedades. Mensualmente se preparan centros de interés para dar visibilidad a los fondos y ofrecer a los usuarios temas de actualidad.

Consulta y Hemeroteca comparten un espacio dotado de amplias mesas, sillones de lectura y grandes ventanales. La Hemeroteca cuenta con una colección de diarios y revistas prestables, organizadas por materias, tanto culturales como de entretenimiento y de actualidad. 


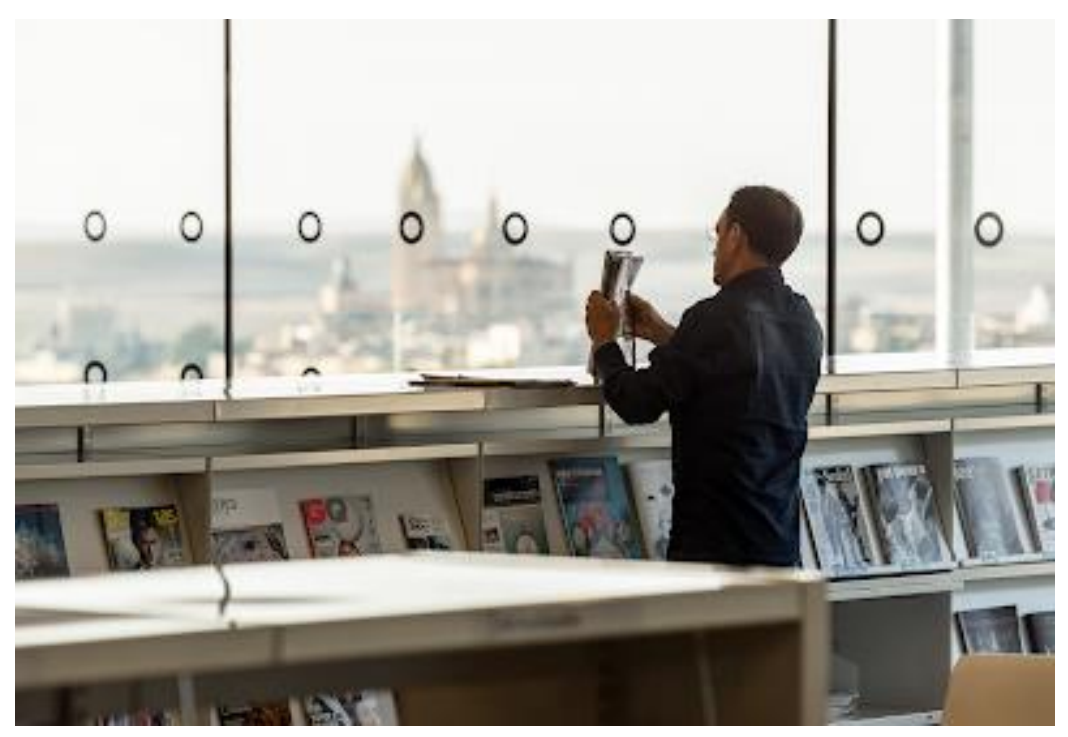

Figura 7: Hemeroteca

La Zona Joven es un espacio independiente, con rincones de lectura y mesas para reunirse. Cuenta con una colección específica de literatura juvenil, álbum ilustrado, cómic y manga. Es el lugar donde se realizan actividades para este público como encuentros informales con autores, talleres y Jornadas del proyecto Acción XXI.

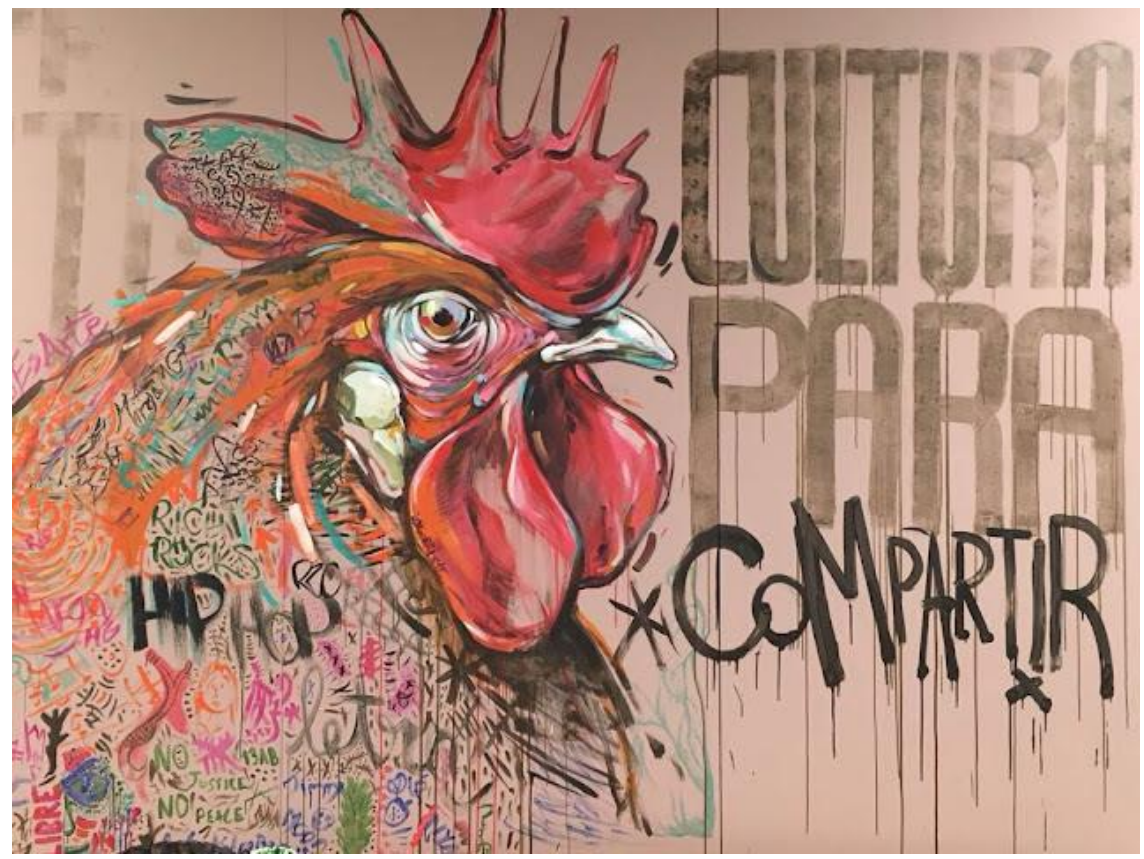

Figura 8: Cultura para todos, intervención dentro del proyecto juvenil Acción XXI

La Sección Infantil es una parte importante de la biblioteca. Cuenta con un recinto independiente en el que está organizada la colección; además dispone de bebeteca y sala de talleres. El movimiento de los fondos en esta sección cobra importancia a través de la preparación de tres 
centros de interés mensuales: efemérides, recomendados y novedades. Así mismo, se realizan actividades de fomento lector todas las semanas.

La Sección Local conserva toda la información de cualquier tipo y en cualquier soporte referente a Segovia y a su provincia: documentos que hablan de arte, de literatura, de economía, de tradiciones, de fiestas, esto es, de cualquier aspecto de la vida de nuestra comunidad.

El Fondo Antiguo se conserva en depósitos, siendo solo accesible para su consulta en sala previa petición. La colección comprende obras desde el siglo XVI, incluyendo algún incunable.

Nuestras colecciones se complementan con los servicios eBiblio, plataforma de lectura y préstamo de libros electrónicos, y cineCYL, visionado en streaming de películas, programas y series de televisión, documentales y cortometrajes.

Además, disponemos de una sala de estudio con capacidad para 80 usuarios y una sala de lectura para investigadores, wifi gratuita y ordenadores con acceso a Internet para adultos y para Infantil con protección, un salón de actos con capacidad para 160 personas, servicio de reprografía y una sala dotada con material para la realización de cursos.

\section{Nuestras redes sociales}

La Biblioteca Pública de Segovia cuenta con varios perfiles en redes sociales para la difusión de sus actividades, la recomendación de libros, música y cines, la realización de concursos y, en general, para visibilizar su tarea de agente cultural:

Twitter@BiblioSegovia

Instagram @bibliotecapublicasegovia

Pinterest @bibsegovia

Youtube BibliotecaPúblicaSegovia

Además, alimentamos dos blog:

Travesías de tinta, con contenidos para adultos a partir de 14 años

Letragones en su tinta, para difusión de la Sección Infantil y de todo lo relacionado con la literatura infantil y juvenil.

El canal donde mostramos nuestro trabajo es la web albergada en el Portal de Bibliotecas de la Junta de Castilla y León, apoyado por la app Biblio JCYL que facilita la gestión de todos los trámites relacionados con los principales servicios de la red: llevar el carné en el móvil, ver los datos del usuario, recibir notificaciones y consultar la agenda de actividades. 
Biblioteca Pública de Segovia

() C/ de los Procuradores de la Tierra, 6. C.P.: 40006. Segovia.

凅 921463533

$\square$ bpsegovia@jcyl.es

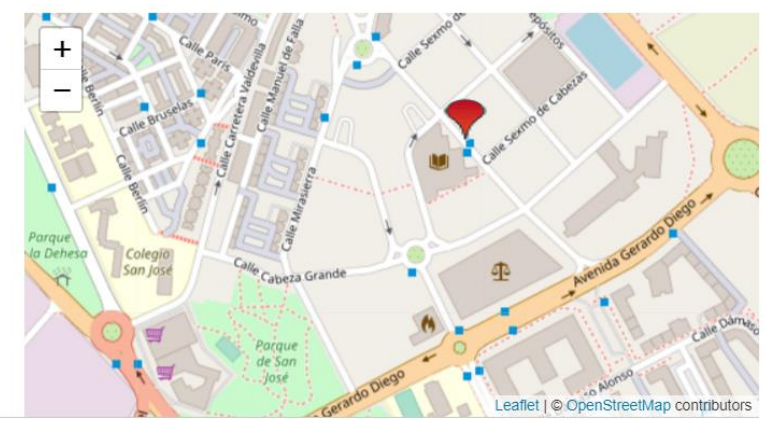

Figura 9: Localización y contacto

\section{Referencias}

De los Reyes Gómez, F. (2021). Desamortización y patrimonio bibliográfico: historia de la Biblioteca Pública de Segovia. Fragua.

Junta de Castilla y León. Anuario estadístico de Castilla y León (2019). https://bibliotecas.jcyl.es/web/jcyl/Bibliotecas/es/Plantilla100/1284743844489/_ _ _

Ministerio de Cultura y Deporte (2011). Proyecto para la nueva sede de la Biblioteca Pública de Segovia https://www.culturaydeporte.gob.es/giec/obras/concluidas/bibliotecas/segovia.html

\section{Sobre la autora}

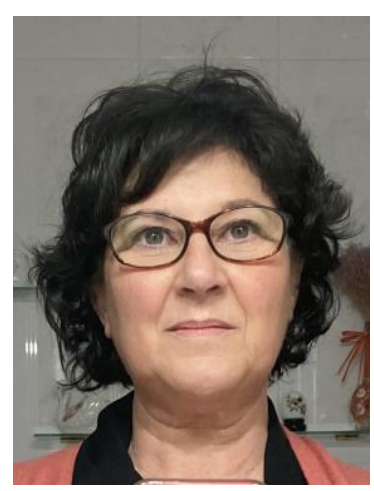

MARÍA LUISA SANTAMARÍA RAMÍREZ

Ayudante de Biblioteca de la Junta de Castilla y León

Licenciada en Filosofía por la Universidad de Salamanca, es Ayudante de Biblioteca en la Biblioteca Pública de Segovia donde coordina las secciones Infantil y Juvenil, Hemeroteca, Equipo de Comunicación y actividades de fomento lector. Desde 2019, Vocal en Junta Directiva de Sedic.santamariaramirez.luisa@gmail.com

- Linkedln

- Twitter 